\title{
EIGENVALUE COMPARISON FOR TUBULAR DOMAINS
}

\author{
JEFFREY M. LEE
}

(Communicated by Jonathan M. Rosenberg)

\begin{abstract}
S. Y. Cheng's eigenvalue comparison theorem for geodesic balls is generalized to certain tubular domains. This gives application to the infinitesimal volume comparison theory developed by $\mathrm{E}$. Heintze and $\mathrm{H}$. Karcher as well as A. Gray.
\end{abstract}

The fundamental tone of an arbitrary open set $U$ contained in a complete Riemannian $n$-manifold $M$ is given by

$$
\lambda^{*}(U)=\inf _{\varphi \in C_{c}^{\infty}(U)} \frac{\int_{U}\langle d \varphi, d \varphi\rangle \omega_{M}}{\int_{U} \varphi^{2} \omega_{M}}
$$

where $\omega_{M}$ is the Riemannian volume form. If $H(U)$ denotes the completion of $C^{\infty}(U)$ in the norm $\|\cdot\|_{1}$ given by

$$
\|f\|_{1}^{2}=\int_{U}\langle d f, d f\rangle \omega_{M}+\int_{M} f^{2} \omega_{M}
$$

and if we denote by $H_{0}(U)$ the completion of $C_{c}^{\infty}(U)$ in $H(U)$ then the infimum in (1) above may be replaced by an infimum over $H_{0}(U)$ with the derivatives being generalized derivatives.

In case $U$ is relatively compact and has sufficiently regular boundary then $\lambda^{*}(U)$ is the first Dirichlet eigenvalue of the Laplacian on $U$. If $B\left(p, r_{0}\right)$ is a geodesic ball in $M$ and $\operatorname{Ric}(X, X) \geq \kappa(n-1)|X|^{2}$ for all $X \in T M$, where Ric denotes the Ricci curvature, then Cheng's comparison theorem [C] states that

$$
\lambda^{*}\left(B\left(p, r_{0}\right)\right) \leq \lambda^{*}\left(B_{\kappa}\left(r_{0}\right)\right),
$$

where $B_{\kappa}\left(r_{0}\right)$ is a geodesic ball in the simply connected space form of curvature $\kappa$ and dimension $n=\operatorname{dim} M$.

In this note we show that the corresponding statement holds for tubular domains of a compact totally geodesic submanifold (e.g. a closed geodesic). We

Received by the editors March 27, 1989 and, in revised form, November 6, 1989.

1980 Mathematics Subject Classification (1985 Revision). Primary 58G25, 53C99; Secondary 35P15, 53C40.

Key words and phrases. Laplace operator, Riemannian manifold, eigenvalues, tubular domain, spectral geometry. 
will also give similar results for general compact submanifolds allowing bounds on the principal curvatures as part of our data. To make this precise we need some notation.

Notation. Let $M$ be an $n$ dimensional complete Riemannian manifold and suppose $P$ is a compact submanifold of dimension $k$. For example, if $k=0$ then $P$ can be any point in $M$. Let $N P \stackrel{\pi}{\longrightarrow} P$ be the normal bundle on $P$ and endow the total space $N P$ with the canonical metric compatible with the normal connection so that vertical vectors are orthogonal to horizontal vectors in $T N P$. Also let $N P_{1}$ denote the unit normal bundle $\left(N P_{1} \subset N P\right)$ with induced metric. Given $\xi \in N P_{1}$ denote by $C(\xi)$ the infimum in $\overline{\mathbb{R}}^{+}=\mathbb{R} \cup\{+\infty\}$ of all $t$, such that $\operatorname{dist}\left(P, \exp _{\pi \xi} t \xi\right)=t$. Thus, $\exp _{\pi \xi} C(\xi) \xi$ is a cut point of $P$ in $M$, and $C: N P_{1} \rightarrow \overline{\mathbb{R}}^{+}$is continuous.

Define a map $\Phi: \mathbb{R}^{+} \times N P_{1} \rightarrow M$ by $(r, \xi) \mapsto \exp _{\pi \xi} r \xi$. $\Phi$ is a diffeomorphism on the set $\left\{(r, \xi) \in \mathbb{R}^{+} \times N P_{1}: 0<r<C(\xi)\right\}$. The Jacobian of this map is $t^{n-k-1} \theta(t \xi)$ where $\theta \omega_{N P}=\exp _{\perp}^{*} \omega_{N}$, in other words, $\theta$ is the Jacobian of the normal exponential map on $N P$.

The type of domains of interest to us are given by

$$
T\left(P, r_{0}\right)=\left\{\exp _{\pi \theta} r \theta \in M: \theta \in N P_{1}, \quad 0 \leq r<r_{0}\right\} .
$$

In case $M=M_{\kappa}^{n}$, the simply connected space form of constant curvature $\kappa$, and $P=S^{k}$, a totally geodesic complete submanifold (isometric to $M_{\kappa}^{k}$ ), we denote the corresponding tubular domain by

$$
T_{\kappa}^{k}\left(r_{0}\right)=T\left(S^{k}, r_{0}\right) \subset \mathbb{M}_{\kappa}^{n} \text {. }
$$

We will prove the following:

Theorem 1. Let $M$ be a complete n-dimensional Riemannian manifold with sectional curvature bounded below by $\kappa$. Let $P$ be a k-dimensional compact totally geodesic submanifold and let $T\left(P, r_{0}\right)$ and $T_{\kappa}\left(r_{0}\right)$ be tubular domains as above. Then we have

$$
\lambda^{*}\left(T\left(P, r_{0}\right)\right) \leq \lambda^{*}\left(T_{\kappa}^{k}\left(r_{0}\right)\right) .
$$

In case $k=0$ or $k=n-1$, we can replace our curvature assumption by the weaker condition

$$
\operatorname{Ric}(X, X) \geq \kappa(n-1)|X|^{2}
$$

where Ric denotes the Ricci curvature.

It is important to notice that $T\left(P, r_{0}\right)$ may not have a regular boundary. The proof below is analogous to the proof of Cheng's comparison theorem [C]. We will use the (infinitesimal) volume comparison theory of $[\mathrm{H}-\mathrm{K}, \mathrm{Gr}]$ for which the curvature assumptions are needed.

Proof. We start by noticing that $\lambda^{*}\left(T_{\kappa}^{k}\left(r_{0}\right)\right)$ is actually an eigenvalue in case $\kappa>0$. The corresponding eigenfunction written in $(r, \xi)$ coordinates is a 
function of $r$ only. This follows since there is a group of isometries of $T_{\kappa}^{k}\left(r_{0}\right)$ preserving each $r$-level set and transitive on each $r$ level set. We will denote this eigenfunction by $\varphi(r)$. Thus, $\varphi$ satisfies

$$
\frac{d^{2} \varphi}{d r^{2}}+\left(\frac{n-k-1}{r}+\frac{d \theta_{\kappa}(r)}{d r} / \theta_{\kappa}(r)\right) \frac{d \varphi}{d r}+\lambda_{\kappa} \varphi=0 .
$$

Here $\theta_{\kappa}$ is the Jacobian of the normal exponential map arising from $S^{k} \subset M_{\kappa}^{n}$ and $\lambda_{\kappa}=\lambda^{*}\left(T_{\kappa}^{k}\left(r_{0}\right)\right)$. However, if $\kappa \leq 0$, then $T_{\kappa}^{k}\left(r_{0}\right)$ is not relatively compact unless $k=0$. Despite this it is not hard to find a $\lambda_{\kappa}$ with $\lambda_{\kappa} \leq \lambda^{*}\left(T_{\kappa}^{k}\left(r_{0}\right)\right)$, and $\varphi(r)$ which satisfies (7) except that now $\varphi$ is not $L^{2}$ for $k>0$.

Now we transfer $\varphi$ to $T\left(P, r_{0}\right)$. Thus we can find a function $F$ satisfying

$$
F(p)=F\left(\exp _{\pi \xi} r \xi\right)=\varphi(r)
$$

where we agree to choose $(r, \xi)$ so that $r<\min \left\{r_{0}, C(\xi)\right\} . F$ is well defined on $T\left(P, r_{0}\right)$ and is continuous since $C(\xi)$ is continuous. Also $\operatorname{grad} F$ is defined continuous and bounded almost everywhere. Now since the boundary of $T\left(P, r_{0}\right)$ may not be regular it is not yet clear that $F \in H_{0}(U)$. To see this let $L:[0, \infty) \rightarrow \mathbb{R}$ be $C^{\infty}$ and such that $L^{\prime}(0)=0$ and supp $L \subseteq\left[0, r_{0}\right)$. Define $G$ on $T\left(P, r_{0}\right)$ by $G\left(\exp _{\pi \xi} r \xi\right)=L(r)$ where $(r, \xi)$ is chosen so that $r<\min \left\{r_{0}, C(\xi)\right\}$ as before. Notice that $G$ is continuous with $\operatorname{grad} G$ bounded and continuous almost everywhere. $G$ also has compact support and hence is in $H_{0}\left(T\left(P, r_{0}\right)\right)$. Now we show that $G$ can be chosen to make $\|F-G\|_{1}^{2}$ as small as we like. Because of the bounds on Ricci curvature and the fact that $P$ is totally geodesic, we can apply the submanifold comparison theory of [H-K ] to conclude that $\theta(r \xi) \leq \theta_{\kappa}(r)$. Thus,

$$
\begin{aligned}
\|F-G\|_{1}^{2} & =\int_{N P_{1}}\left(\int_{0}^{\min \left\{C(\xi), r_{0}\right\}}\left[(\varphi-L)^{2}+|\nabla \varphi-\nabla L|^{2}\right] r^{n-k-1} \theta(r \xi) d r\right) \omega_{N P_{1}} \\
& \leq \int_{N P_{1}} \omega_{N P_{1}} \int_{0}^{r_{0}}\left[(\varphi-L)^{2}+\left(\frac{d \varphi}{d r}-\frac{d L}{d r}\right)^{2}\right] r^{n-k-1} \theta_{\kappa}(r) d r,
\end{aligned}
$$

where $\omega_{N P_{1}}$ is the Riemannian volume form on $N P_{1}$. This last term can be made as small as we like by standard considerations. Thus, $F \in H_{0}(U)$.

Let $b(\xi)=\min \left\{r_{0}, C(\xi)\right\}$. Now to complete the proof we only need to show that

$$
\int_{0}^{b(\xi)}\left(\frac{d \varphi}{d r}\right)^{2} r^{n-k-1} \theta(r \xi) d r \leq \lambda_{\kappa} \int_{0}^{b(\xi)} \varphi^{2} r^{n-k-1} \theta(r \xi) d r
$$

as integration over $\xi \in N P_{1}$ shows. Upon integration by parts, the left side of 
the above inequality becomes

$$
\left.\varphi \frac{d \varphi}{d r} \theta(r \xi)\right|_{0} ^{b(\xi)}-\int_{0}^{b(\xi)} \frac{\varphi}{r^{n-k-1} \theta(r \xi)} \frac{d}{d r}\left(r^{n-k-1} \theta(r \xi) \frac{d \varphi}{d r}\right) r^{n-k-1} \theta(r \xi) d r .
$$

We can assume $\varphi \geq 0$ and then it is not hard to show that $d \varphi / d r<0$ on $\left(0, r_{0}\right)$. But the comparison theory of $[\mathrm{H}-\mathrm{K}]$ (see $\S 3.4 .1$ ) also gives us $\frac{d \theta(r \xi)}{d r} / \theta(r \xi) \leq \frac{d \theta_{\kappa}}{d r} / \theta_{\kappa}$ so that

$$
\begin{aligned}
\frac{1}{r^{n-k-1} \theta(r \xi)} & \frac{d}{d r}\left(r^{n-k-1} \theta(r \xi) \frac{d \varphi}{d r}\right) \\
& =\frac{d^{2} \varphi}{d r^{2}}+\left(\frac{n-k-1}{r}+\frac{d \theta(r \xi)}{d r} / \theta(r \xi)\right) \frac{d \varphi}{d r} \\
& \geq \frac{d^{2} \varphi}{d r^{2}}+\left(\frac{n-k-1}{r}+\frac{d \theta_{\kappa}}{d r} / \theta_{\kappa}\right) \frac{d \varphi}{d r}=-\lambda_{k} \varphi .
\end{aligned}
$$

Hence,

$$
\begin{aligned}
\int_{0}^{b(\xi)}\left(\frac{d \varphi}{d r}\right)^{2} r^{n-k-1} \theta(r \xi) d t \leq & \left.\varphi \frac{d \varphi}{d r} r^{n-k-1} \theta(r \xi)\right|_{0} ^{b(\xi)} \\
& +\int_{0}^{b(\xi)} \lambda_{\kappa} \varphi^{2} r^{n-k-1} \theta(r \xi) d r \\
& \leq \lambda_{\kappa} \int_{0}^{b(\xi)} \varphi^{2} r^{n-k-1} \theta(r \xi) d r
\end{aligned}
$$

where we have used $d \varphi / d r \leq 0$. This completes the proof.

In case the submanifold $P$ is not totally geodesic we can still obtain some information by this method, provided that $r_{0}$ is not too large. Given $\xi \in$ $N P_{1}$, let $S_{\xi}$ be the associated shape operator on $T_{\pi \xi} M$ defined by $\left\langle S_{\xi} X, Y\right\rangle=$ $\left\langle\nabla_{X} \bar{\xi}, Y\right\rangle$ where $\bar{\xi}$ is a normal field extending $\xi$. Let $\mu_{1}(\xi), \ldots, \mu_{k}(\xi)$ denote the eigenvalues of $S_{\xi}$ and define $\mu$ by

$$
\mu=\sup _{i, \xi}\left\{\left|\mu_{i}(\xi)\right|\right\} .
$$

Define functions $\theta_{\xi}(\kappa, r)$ and $\Theta_{\mu}(\kappa, r)$ as follows:

$$
\theta_{\xi}(\kappa, r)=\left(\mathbf{s}_{\kappa}(r)\right)^{n-k-1} \operatorname{det}\left(\mathbf{c}_{\kappa}(r) I+\mathbf{s}_{\kappa}(r) S_{\xi}\right)
$$

and

$$
\Theta_{\mu}(\kappa, r)=\left(\mathbf{s}_{\kappa}(r)\right)^{n-k-1}\left(\mathbf{c}_{\kappa}(r)+\mu \mathbf{s}_{\kappa}(r)\right)^{k}
$$

where

$$
\mathbf{s}_{\kappa}(r)=\left\{\begin{array}{lll}
(1 / \sqrt{\kappa}) \sin \sqrt{\kappa} r & \text { if } & \kappa>0 \\
r & \text { if } & \kappa=0 \\
1 / \sqrt{|\kappa|} \sinh \sqrt{|\kappa|} r & \text { if } & \kappa<0
\end{array}\right.
$$

and $\mathbf{c}_{\kappa}(r)=\mathbf{s}_{\kappa}^{\prime}(r)$. 
If we assume that the sectional curvature of $M$ is bounded below by $\kappa$, then the comparison theory of [Gr] (cf. proof of Lemma 6.2) gives $\theta(r \xi) \leq \theta_{\xi}(\kappa, r)$ and $\frac{d}{d r} \ln (\theta(r \xi)) \leq \frac{d}{d r} \ln \left(\theta_{\xi}(\kappa, r)\right)$. However, a straightforward calculation also shows that for $r \geq 0$, and in case $\kappa>0, r \leq \pi / 2 \sqrt{\kappa}$ we have $\theta_{\xi}(\xi, r) \leq$ $\Theta_{\mu}(\kappa, r)$ and $\frac{d}{d r} \ln \theta_{\xi}(\kappa, r) \leq \frac{d}{d r} \ln \Theta_{\mu}(\kappa, r)$. Thus we have

$$
\theta(r \xi) \leq \Theta_{\mu}(\kappa, r)
$$

and

$$
\frac{d}{d r} \ln \theta(r \xi) \leq \frac{d}{d r} \ln \Theta_{\mu}(\kappa, r) .
$$

Now we define $\lambda\left(\kappa, \mu, r_{0}\right)$ to be the first eigenvalue of the following (mixed) eigenvalue problem on $\left[0, r_{0}\right]$.

$$
\frac{d^{2} \varphi}{d r^{2}}+\left(\frac{n-k-1}{r}+\frac{d \Theta_{\mu}(\kappa, r)}{d r} / \Theta_{\mu}(\kappa, r)\right) \frac{d \varphi}{d r}+\lambda \varphi=0,
$$

$\varphi^{\prime}(0)=0, \quad \varphi\left(r_{0}\right)=0$.

Theorem 2. Let $M$ be a complete n-dimensional Riemannian manifold with sectional curvature bounded below by $\kappa$. Let $P$ be a $k$-dimensional compact Riemannian submanifold of $M$. Given $r_{0} \geq 0$, in case $\kappa>0, r_{0} \leq \pi / 2 \sqrt{\kappa}$ let $T\left(P, r_{0}\right)$ be a tubular domain defined as in (3). Assume that $\mu$ is a bound on the absolute value of the principle curvatures given as in (5). Then we have

$$
\lambda^{*}\left(T\left(P, r_{0}\right)\right) \leq \lambda\left(\kappa, \mu, r_{0}\right),
$$

where $\lambda\left(\kappa, \mu, r_{0}\right)$ is the lowest eigenvalue of (8) above.

Proof. The proof is formally the same as the proof of Theorem 1 except that $\theta_{\kappa}(r)$ is replaced by $\Theta_{\mu}(\kappa, r)$ and $\lambda_{\kappa}$ by $\lambda\left(\kappa, \mu, r_{0}\right)$. At the two steps where comparison theory of $[\mathrm{H}-\mathrm{K}]$ is used we use (6) and (7) instead.

Added in proof. It has been pointed out to the author that the ideas contained in Michael Gage's paper, Upper bounds for the first eigenvalue of the LaplaceBeltrami operator, Indiana Univ. Math. J. 29 (1980), 897-912, overlap those of the present paper. However, this paper differs somewhat in point of view from Professor Gage's paper in that we do not give formulas for upper bounds on $\lambda_{1}$ but rather compares eigenvalues, concentrating on those cases which are most directly analogous to the comparison results of S. Y. Cheng. Also, even though tubes as such are not the topic of Professor Gage's paper, interesting upper bounds for the first eigenvalue of tubes may be obtained from his results. The reader is advised to consult this work.

The author would like to thank Professor Gage for his graciousness in this matter. 


\section{REFERENCES}

[C] S.-Y. Cheng, Eigenvalue comparison theorems and its geometric applications, Math. Z. 143 (1975), 289-297.

[Gr] A. Gray, Comparison theorems for the volumes of tubes as generalizations of the Weyl tube formula, Topology 21 (1982), 201-228.

[H-K] E. Heintze and H. Karcher, A general comparison theorem with applications to volume estimates for submanifolds, Ann. Sci. Ecole Norm. Sup. 11 (1978), 451-470.

Department of Mathematics, Purdue University, West Lafayette, Indiana 47907 